\title{
TLR family gene expression in relation to the HIF $1 a$ and the VEGFR pathway activation in endometrial cancer
}

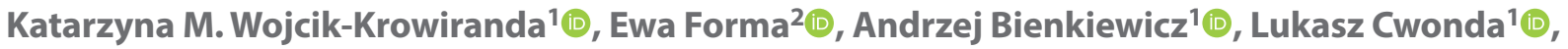 \\ Joanna Wronska-Stefaniak ${ }^{1}$, Magdalena Brys ${ }^{2}{ }^{\circledR}$ \\ ${ }^{1}$ Clinical Department of Gynecologic Oncology, Medical University of Lodz, Poland \\ ${ }^{2}$ Department of Biology, University of Lodz, Poland
}

\begin{abstract}
Introduction: Malignant neoplasm of the endometrium is the most common malignant neoplasm of the female reproductive system. Toll Like Receptors (TLR) play a significant role in innate and late-immunity against infections or damaged tissues. TLRs are also involved in the development of tumors in their natural microenvironment. TLRs play an important role in angiogenesis, necessary for survival and growth of the tumor. Hypoxia playing a critical role in angiogenesis, carcinogenesis, tumor progression and distant metastasis is primarily mediated through hypoxia inducible factors (HIFs). Vascular endothelial growth factor family proteins (VEGF) are also strongly involved in tumor angiogenesis and their action is strongly associated with TLR receptors.

Objectives: The aim of the study was to correlate the expression of selected TLRs and VEGFR's as well as HIF1a with clinicopathological data of endometrial cancer patients.

Material and methods: 123 neoplastic endometrial samples were included in the study. 51 samples of healthy endometrium served as control. The expression of TLR1, TLR2, TLR3, TLR4, VEGFR1 and VEGFR2, VEGF-A and HIF1a was examined after RNA isolation at the mRNA level by Real Time-PCR.

Results: We have noted a significant correlation between the expression of selected TLR and VEGFR's and clinical stage as well as pathological grading of endometrial cancer.

Conclusions: Received correlations confirm a significant contribution of some TLR expression and the receptor for VEGF in the pathogenesis of epithelial endometrial cancer.
\end{abstract}

Key words: endometrial cancer; TLR family; VEGF; VEGR2; VEGFR1; HIF1-a

Ginekologia Polska 2020; 91, 8: 439-446

\section{INTRODUCTION}

Endometrial carcinoma is the most common malignant neoplasm of the female reproductive system worldwide. The increasing number of cases is related to the increasing number of women with endometrial cancer risk factors, both in Poland and in other developed countries.

Mechanisms regulating the carcinogenesis as well as the cell death process are not fully understood.

The solid tumor growth and its metastatic ability depends mainly on the angiogenesis. Thus, the process of tumor vessels growing is a promising therapeutic target. In recent years, important progress in molecular targeted therapy, including antiangiogenetic therapy has been observed. The issue limiting the efficacy of this kind of therapy is the drug resistance. Therefore, further efforts to better understand and eliminate this resistance are required.
In solid tumors the area of low oxygen tension, significantly hypoxic when compared to the healthy tissue are observed. The solid tumor formation is accompanied by local hypoxia, which is often considered to be an independent prognostic factor in many malignancies [1]. Hypoxia-inducible factor-1 (HIF-1) induced in the low oxygen tumor region is known to be an important transcription factor, mediating the cellular activity in the low oxygen environment. Since the mid-eighties, research on their potential participation in the regulation of the cancer process is ongoing.

Toll-like receptors (TLRs) are type I transmembrane receptors that are involved in the recognition and transmission of pathogens to the immune system. They also play an important role in tissue homeostasis. TLRs are a transmitter of information about damaged tissues, which may play a role in the phenomenon of cancer. TLRs are 
a family of particles that recognize the structure of ligands derived from microorganisms or damaged host cells. Their name is related to their similarity to the protein coded by the Toll gene identified in Drosophila [2]. Binding of ligand and TLR plays a key role in innate and late immunity $[3,4]$. The TLR family consists of at least 13 types [5]. Eleven of these (TLR1 to TLR11) have so far been identified in humans. They are located on the surface or in the cytoplasm of immune cells and recognize various molecules and molecular products (DAMP and PAMP). PAMPs are molecular products derived from pathogens. DAMPs are endogenous molecules released from damaged or dying cells. Both DAMP-dependent and PAMP-dependent immune responses via TLR signals are known [6]. TLRs are also involved in the development of tumors in their natural microenvironment. The tumor microenvironment, including cancer cells, normal cells subjected to the "stressogenic" factor, stromal tissue and extracellular matrix, have recently been recognized as the main factor in the progression and metastasis of cancer [7]. Lowering the level of anti-cancer antibodies is the cause of decreased activity of infiltrating immune cells, resulting in cancer progression, angiogenesis and metastasis [8, 9]. Recent studies show that activated TLRs on tumor cells can suppress the anti-tumor effect and functions of infiltrating immune cells, thus altering the inflammatory response in a way that promotes tumor growth [10]. Epithelial cells of the female reproductive system can undergo neoplastic transformation due to continuous TLR stimulation by PAMP. Four types of TLR (TLR2-5) were expressed in ovarian cancer cell lines [11]. Activation of TLR4 promotes the survival of ovarian cancer cells by inducing the expression of antiapoptotic proteins [12]. It was also shown that TLR5 and TLR9 may contribute to the development of cervical cancer $[13,14]$. It seems that TLRs play an important role in angiogenesis necessary for survival and growth of the tumor. The main factor is vascular endothelial growth factor (VEGF) - involved in tumor angiogenesis and associated with TLR signals. Vascular endothelial growth factor (VEGF-A) is a key molecule involved in the process of angiogenesis. [15]. Over-expression of VEGF in tumor cells enhances tumor growth and metastasis in several malignancies, including endometrial cancer. The structure of new vessels, induced by VEGF, is different from the one of normal vessels. Their different permeability leads to high interstitial pressure and further hypoxia, which stimulates additional VEGF production [16]. Solid tumors due to the characteristic hypoxia, which is a stress factor leading to the emergence and release of DAMP [17]. These ligands activate TLR signals and contribute to molecular abnormalities in the tumor microenvironment. However, under tumor conditions, the cells die through non-apoptotic pathways, mainly necrosis. DAMPs released from damaged or dying cells are recognized by TLR on immune cells; subsequent disturbances of the signal recognized by TLR lead to the progression of cancer [18].

The few data available in the literature refer to the potential contribution of individual TRLs to tumorigenesis in endometrial cancer.

\section{MATERIAL AND METHODS}

A total of 123 women with uterine endometrial endometrioid cancer were enrolled to this study. All patients included in the present study gave their written informed consent. Post-operative tissues were subjected to routine histopathological examination in which the histological type of the tumor, the clinical stage of the tumor according to FIGO, the degree of cellular differentiation (grading), tumor size, lymph node status, involvement of surrounding tissues - ovaries, fallopian tubes, cervix, parametria - were assessed. The control group consisted of 51 samples of healthy endometrium taken from surgically removed uteri for non-oncological reasons. Immediately after uterus resection, approx. $0.2 \mathrm{~g}$ cancer tissue samples, (from visible part of the tumor), removed from the uterus, were placed in RNAlater (Ambion, USA) for overnight incubation. Samples were subsequently stored at $-80^{\circ} \mathrm{C}$ until RNA extraction. Clinico-pathological and demographic data of patients are presented in Table 1.

\section{Methods \\ RNA extraction and CDNA synthesis}

Total RNA was isolated from frozen samples of tissue using EXTRACTME Total RNA Kit (Blirt, Poland) according to manufacturer's protocol. Quantity and quality of the isolated RNA were assessed spectrophotometrically. First strand CDNAs were obtained by reverse transcription of $2 \mu \mathrm{g}$ of total RNA using High Capacity cDNA Revers Transcription Kit (Life Technology, USA) following the manufacturer's protocol.

\section{Quantitative real time $P C R$ analysis}

The relative expression levels of $T L R 1, T L R 2, T L R 3$, TLR4 and VEGFR1 were analyzed by real time PCR using the TaqMan Gene Expression Assays (Life Technology, USA) according to manufacturer's instruction. Quantitative data were normalized to HPRT1 used as reference gene. The assays numbers for studied genes were as follows $\mathrm{Hs} 00413978$ m1 — TLR1,Hs02621280_s1 - TLR2, Hs01551078_m1 - TLR3,

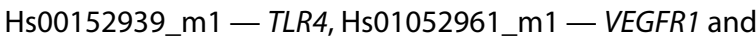
Hs02800695_m1 (reference gene).

Each PCR reaction was performed in duplicate and included $1 \mu$ l of CDNA, $3.5 \mu$ l water, $5 \mu$ l of $2 x$ TaqMan ${ }^{\circ}$ Universal PCR MasterMix (Life Technology, USA) and $0.5 \mu$ l of TaqMan ${ }^{\circ}$ 


\begin{tabular}{|c|c|c|}
\hline & study group n (\%) & control group n (\%) \\
\hline group size & $\mathrm{n}=123$ & $n=51$ \\
\hline age, the average age & $68.50 \pm 10.32$ & \\
\hline$<50$ years & $7(5.69)$ & \\
\hline$\geq 50$ years & $116(94.31)$ & \\
\hline \multicolumn{3}{|c|}{ clinical-pathological characteristics pT } \\
\hline pT1-pT2 & $112(91.0)$ & \\
\hline pT3-pT4 & $11(9.0)$ & \\
\hline \multicolumn{3}{|l|}{$\mathrm{pN}$} \\
\hline pNO & $98(79.67)$ & \\
\hline pN1-pN3 & $19(15.44)$ & \\
\hline no data & $6(4.89)$ & \\
\hline \multicolumn{3}{|l|}{ G } \\
\hline G1 & $12(9.75)$ & \\
\hline G2 & $84(68.29)$ & \\
\hline G3 & 17 (13.82) & \\
\hline no data & $10(8.14)$ & \\
\hline \multicolumn{3}{|l|}{ FIGO } \\
\hline |-II & $101(82.1)$ & \\
\hline III-IV & $21(17.07)$ & \\
\hline no data & $1(0.83)$ & \\
\hline \multicolumn{3}{|c|}{ myometrium infiltration } \\
\hline$<1 / 2$ & $62(50.04)$ & \\
\hline$>1 / 2$ & $58(47.15)$ & \\
\hline no data & $3(2.81)$ & \\
\hline
\end{tabular}

Gene Expression Assays consisted of a pair unlabeled PCR primer and a TaqMan ${ }^{\circ}$ probe with FAM $^{\mathrm{TM}}$ dye label on the $5^{\prime}$ end and MGB nonfluorescent quencher on the $3^{\prime}$ end. The following PCR program was used: $95^{\circ} \mathrm{C}$ for $10 \mathrm{~min}, 40$ cycles of $95^{\circ} \mathrm{C}$ for $15 \mathrm{~s}, 1 \mathrm{~min}$ annealing and extension at $60^{\circ} \mathrm{C}$. PCR reactions were carried out using the Mastercycler ep realplex (Eppendorf, Germany).

The equation $2^{-\Delta C t}$ was applied to calculate the expression of studied genes, where $\Delta \mathrm{Ct}=\mathrm{Ct}$ of the target gene - $\mathrm{Ct}$ the reference gene (HPRT1). Results are expressed as a number of target gene mRNA copies per 1000 copies of HPRT1 mRNA.

The relative expression levels of VEGFR2, VEGF and HIF-1a were analyzed by real time PCR using SYBR Green reagent (Applied Biosystems, USA) according to Luczak et al., [1] and Amirchaghmaghi et al., (2015).

\section{Statistical analysis}

Statistical analysis was performed using PQStat version 1.6.4 (PQStat Software, Poland). Differences of mRNA expression among groups were analyzed by non-parametric test (Mann-Whitney $U$ test and Kruskal-Wallis test with post hoc multiple comparisons). Co-expression of genes was analyzed using the Spearman test. A value of $p<0.05$ was considered statistically significance.

\section{RESULTS}

The expression of TLR1, TLR2, TLR3, TLR4, VEGFR1 VEGFR2, VEGF-A and HIF-1a at the mRNA level were correlated to clinical and pathological features.

\section{Tumor size (pT)}

\section{a) TLR1 gene}

The lowest values of the TLR1 gene were recorded in the T3-T4 endometrial cancer group. This difference was statistically significant when compared to the control $(p<0.002)$ (Fig. 1).

\section{b) TLR2 gene}

There were no statistically significant differences between the examined groups.

\section{c) TLR3 gene}

There were highly significant differences between the examined groups. Both T1-T2 and T3-T4 groups revealed significantly lower expression of TLR3 $(p<0.0001)$ when compared to the control group (Fig. 1).

\section{d) TLR4 gene}

There were no statistically significant differences between TLR4 gene expression in the examined groups.

\section{e) VEGFR1 gene}

The VEGFR1 gene expression in the control group was significantly lower than in the group of patients with small (T1-2) and large (T3-4) tumors ( $p<0.003$ and $p<0.0001$, respectively). VEGFR1 expression for T1- T2 group did not differ from the T3-T4 group (Fig. 1).

\section{f) VEGFR2 gene}

The VEGFR2 gene expression in the control group was significantly higher than in the group of patients with small (T1-2) and large (T3-4) tumors ( $p 0.0001$ and $p<0.0001$, respectively). VEGFR2 expression for T1- T2 group did not differ from the T3-T4 group (Fig. 1).

\section{g) VEGF-A gene}

There were no statistically significant differences between the examined groups.

\section{h) HIF1a}

The significantly lower expression of HIF1a was observed only in a T1-T2 group when compared to the control (Fig. 1).

\section{Lymph node status (pN)}

\section{a) TLR1 gene}

The highest TLR1 expression was found in the control group. They differed significantly from the lowest values found in the group of patients with endometrial cancer without regional lymph node involvement $(p<0.05)$. There were no other significant differences in TLR1 expression between the examined groups (Fig. 2). 

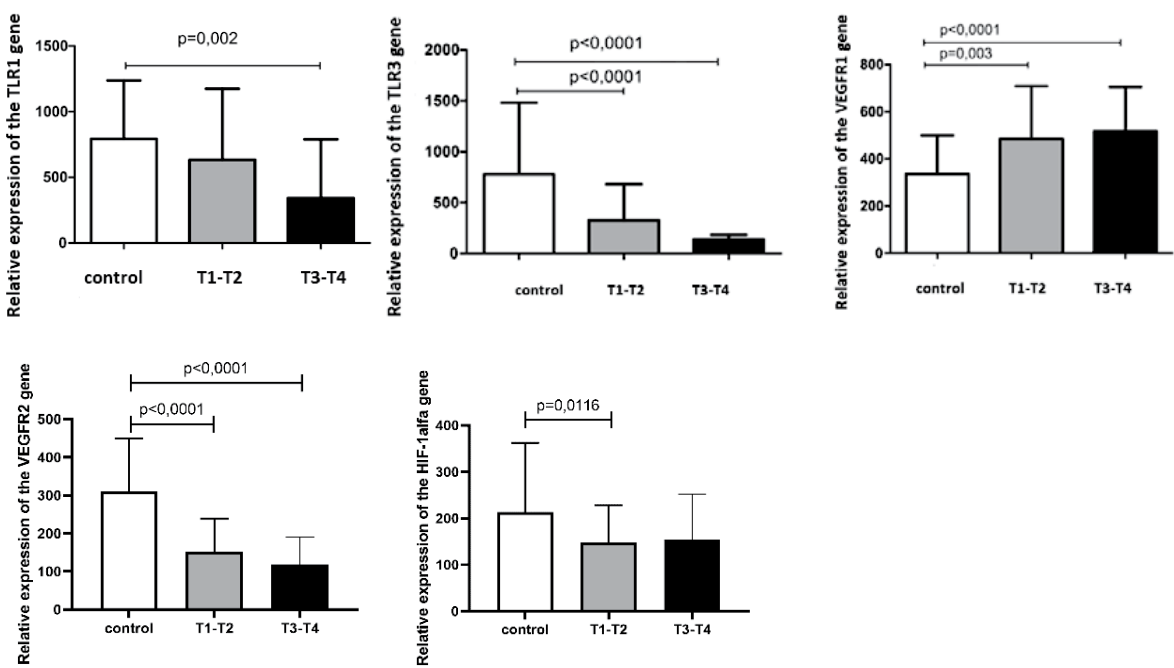

Figure 1. Expression of the TLR, VEGFR and HIF-1alfa genes depending on the size of the tumor
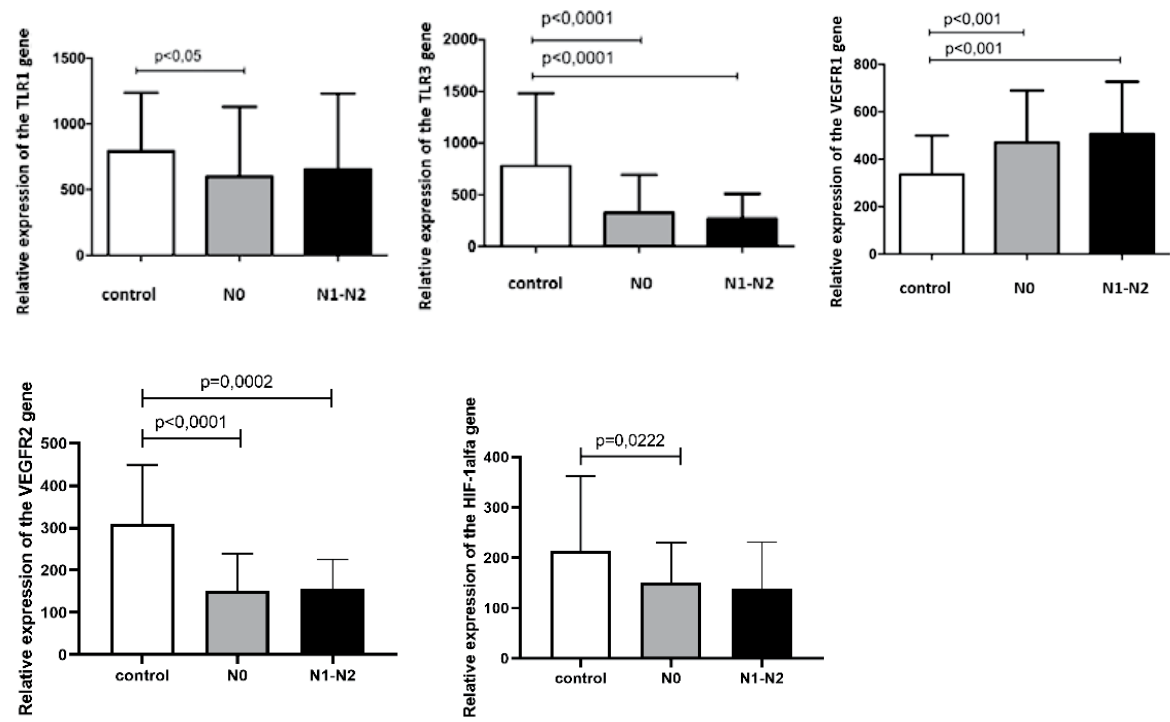

Figure 2. Expression of the TLR, VEGFR and HIF-1alfa genes depending on the state of regional lymph nodes

\section{b) TLR2 gene}

The expression of the TLR2 gene did not differ statistically between the control group and the group of patients with endometrial cancer regardless of nodal status.

c) TLR3 gene

TLR3 gene expression was significantly lower (at the same level of significance $-p<0.0001$ ) in both endometrial cancer groups, i.e. regardless of the lymph node status, when compared to the control group (Fig. 2).

d) TLR4 gene

There were no statistically significant differences between TLR4 gene expression in the examined groups compared to the control group.

\section{e) VEGFR1 gene}

The VEGFR1 gene expression was significantly higher $(p<0.0001)$ in the group of women with cancer regardless of the lymph node status, when compared to the control (Fig. 2).

f) VEGFR2 gene

The VEGFR2 gene expression in the control group was significantly higher $(p<0.0001-0.0002)$ than in the cancer groups regardless of the lymph node status, when compared to the control (Fig. 2).

g) VEGF-A gene

There were no statistically significant differences between the examined groups.

h) HIF1a 

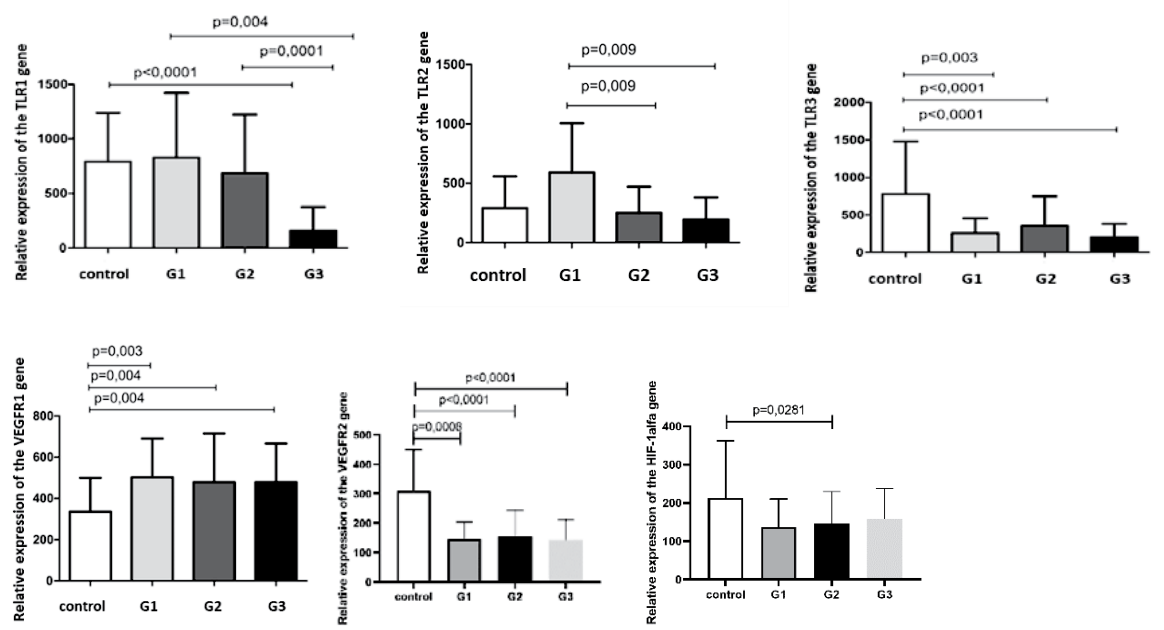

Figure 3. Expression of the TLR, VEGFR and HIF-1alfa genes depending on the grade of malignancy of the tumor

The significantly lower $(\mathrm{p}<0.022)$ expression of HIF1a was observed only in a NO group, when compared to the control (Fig. 2).

\section{Tumor differentiation (G)}

\section{a) TLR1 gene}

The lowest values of TLR1 gene expression were recorded in low-grade tumors (G3). This difference showed a high statistical significance $(p<0.0001)$ when related to $\mathrm{G} 1, \mathrm{G} 2$ and control group. TLR1 expression in groups $\mathrm{G} 2$ and G1 did not differ significantly from the control (Fig. 3).

\section{b) TLR2 gene}

The highest TLR2 gene expression values were observed in $\mathrm{G} 1$ tumors. Significant differences were found between well differentiated tumors (G1) in comparison to G2 and G3 tumors. However, no statistically significant differences were found between the studied groups and the control (Fig. 3).

c) TLR3 gene

TLR3 gene expression is lower in all examined cancer groups regardless of the degree of tumor differentiation (G) when compared to the control. For all G groups, those relations were highly $(p<0.003, p<0.0001)$ different (Fig. 3).

d) TLR4 gene

Expression of the TLR4 gene did not differ statistically between the examined cancer groups when compared to the control.

e) VEGFR1 gene

VEGFR1 gene expression was significantly higher in all examined cancer groups, regardless of the tumor differentiation $(G)$ when compared to the control group. For all $\mathrm{G}$ degrees, these relations were characterized by high statistical significance ( $p<0.003, p<0.004)$ (Fig. 3).

f) VEGFR2 gene

VEGFR2 gene expression was significantly lower in all examined cancer groups, regardless of the tumor dif- ferentiation $(G)$ when compared to the control group. For all $\mathrm{G}$ degrees, these relations were characterized by high statistical significance ( $p<0.0001, p<0.0001$ and $\mathrm{p}<0.0008$ ) (Fig. 3).

g) VEGF-A gene

There were no statistically significant differences between the examined groups.

h) HIF1a

The significantly lower expression of HIF1a was observed only in G2 group when compared to the control (Fig. 3).

\section{FIGO stage of endometrial cancer}

\section{a) TLR1 gene}

There were no statistically significant differences in TLR1 gene expression related to FIGO stage between the examined groups and the control.

b) TLR2 gene

There were no statistically significant differences in the expression of the TLR2 gene between the examined groups and the control.

c) TLR3 gene

In the examined groups of patients with endometrial cancer, the expression of the TLR3 gene was significantly lower, regardless of the FIGO stage, when compared to the control group ( $p<0.0001$ ) (Fig. 4).

d) TLR4 gene

There were no statistically significant differences in TLR4 gene expression between the examined groups and the control.

e) VEGFR1 gene

VEGFR1 gene expression was significantly higher $(p<0.0001)$ in the examined groups when compared to the control group, regardless of the FIGO stage (Fig. 4).

f) VEGFR2 gene 

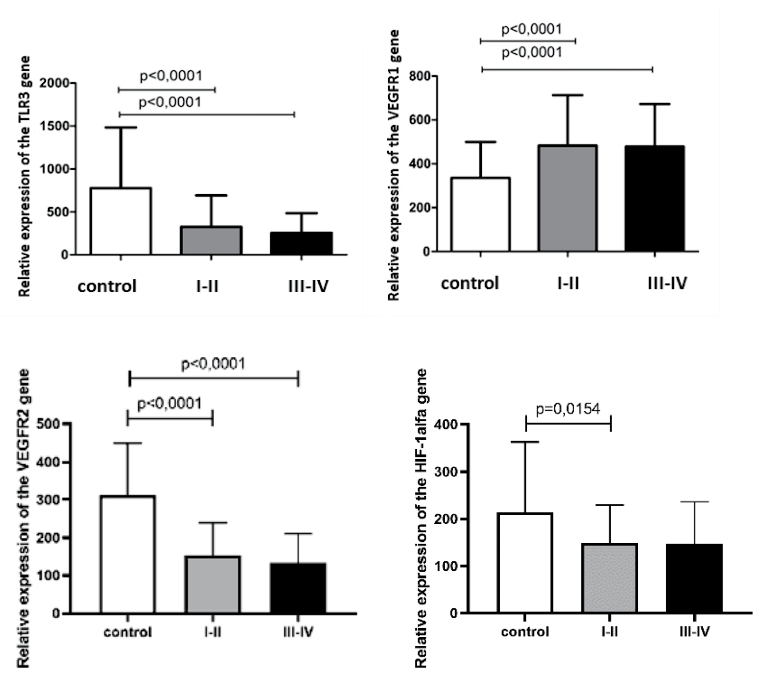

Figure 4. Expression of the TLR, VEGFR and HIF-1alfa genes depending on the FIGO stage

In the examined groups of patients with endometrial cancer, the expression of the VEGFR2 gene was significantly lower, regardless of the FIGO stage, when compared to the control ( $p<0.0001)$ (Fig. 4).

g) VEGF-A gene

There were no statistically significant differences between the examined groups.

h) HIF1a

The significantly lower $(p<0.0154)$ expression of HIF1a was observed only in FIGO I-Il group when compared to the control (Fig. 4).

\section{DISCUSSION}

TLRs are a link that combines non-specific with specific immunity. Their role in the pathogenesis of malignant tumors is not fully understood. In the available literature, there are few data about the TRL receptor compound, with mechanisms related to cancer and the spread of tumors in the human body. It is known that the expression of certain types of TLRs is different in cancers of the ovary, cervix, large intestine, breast and others [10]. There are also some data on participation of the TLRs in physiological processes in the endometrium, as well as in endometrial hypertrophy and some post-partum pathologies [19]. Hypoxia playing a critical role in angiogenesis, carcinogenesis, tumor progression and distant metastasis is primarily mediated through hypoxia inducible factors (HIFs) [20]. In the available literature, however, there are few reports analyzing the potential importance of TLRs in the pathogenesis of endometrial cancer and its relation to specific hypoxia markers.

In our study, we correlated the expression of selected TLRs with angiogenic and hypoxia factors in relation to the clinico-pathological data.
Expression of TLR3 was significantly lower, regardless of the size of the tumor, represented in the pathomorphological classification as a T feature, in relation to the control. In turn, TLR1 receptor expression only in advanced (T3-T4) tumors, similarly as TLR3, was significantly reduced. These data are partly correlated with the report by Allhorn et al. [21], who evaluated the expression of TLR3 and TLR4 in various conditions of the endometrium and revealed, among others, that TLR3 and TLR4 expression is significantly reduced in low-differentiated endometrial tumors. However, authors did not refer those results to the clinico-pathological data of the disease. In contrast to the above results, we did not demonstrate the important role of the TRL4 receptor in the pathogenesis of endometrial cancer.

In turn, according to expectations and data from the literature [16, 22], the expression of VEGFR1 was significantly increased in cancer tissue, when compared to the control, irrespective of their size, grading, nodal status and FIGO stage. In contrast, the VEGFR2 expression was significantly decreased in the endometrial cancer samples in the tumor size independent manner.

The relations between transcriptome and proteome is not a clear and direct issue. The protein and mRNA cellular content might be influenced by many factors. One can suppose, the mRNA expression may be regulated by the negative feedback control mechanism. The protein to protein interactions may stabilize some proteins. This can be induced by protein's physiological turnover disruption.

Protein halflive increases due to its stabilisation what is induced when components involved in normal protein's turnover are disrupted or through protein to protein interactions. This phenomenon may occur when downregulation of mRNA and simultaneous protein upregulation are observed.

Akin to VEGFR1, VEGFR2 expression was related to the presence of malignancy regardless of tumor differentiation, nodal status and FIGO stage. This observation indirectly confirms the important role of VEGF receptors in the development of endometrial cancer.

In our study we were unable to demonstrate correlation between VEGF-A protein and clinico pathological data of examined tissues.

The presence of metastases in the lymph nodes did not influence on TLR3 expression. Significantly lower TLR3 expression was observed in cancerous tumors irrespective to the lymph node status.

Tumor malignancy $(G)$ is one of the most important, independent prognostic factors in endometrial cancer. We have demonstrated the highly significant correlation between cell differentiation and some TLRs, VEGFR1, VEGFR2 expression. Like in other clinic-pathologic factors, the expression of VEGFR1 and VEGFR2 were opposite when related to the tumor malignancy. 
In undifferentiated tumors (G3), TLR3 expression was significantly lower than in the control group. It was also decreased in comparison to more differentiated tumors (G2 and G1), but this difference was not statistically significant. Similar results were obtained by Allhorn et al. [21]. Although the results given in the cited work were based on the analysis of only 16 cases of endometrial cancer, similarly to our results, the authors observed significantly lower TLR3 expression in all degrees of cellular differentiation. Different results on TLR4 expression in endometrial cancer tissue were presented by Allhorn et al. In our study, the expression of this receptor in tumor tissues did not differ from the control group, while Allhorn observed a relationship between TLR4 expression and tumor differentiation, like that found in TLR3 results.

Similarly, VEGF1 and VEFGR2 receptors expression were different in neoplastic tumors irrespective to the regional nodal involvement. This highly significant correlation, negative for VEGFR1 and positive for VEGRF2 seems to support the hypothesis of their direct involvement in cancerogenesis [23]. Similar results were obtained by [24] and [25].

Gene expression for VEGFR1 appears to be inversely related to TLR3 expression. Similar to the available data [18], in all malignant endometrial tumors, it was significantly higher than in the control group. Analogous relations were observed by Giatromanolaki A et al. [26] who demonstrated that VEGFR expression is one of significant independent prognostic factors in epithelial endometrial tumors. In contrast, gene expression of VEGFR2 appeared to be directly related to TLR3 expression. The explanation of those differences needs further studies.

Surprisingly, Hypoxia Inducible Factor-1a (HIF-1a) supposed to play a key role in hypooxygenation of cancer tissue was not overexpressed in endometrial cancer tissue in more advanced, undifferentiated tumors.

We have not found a highly significant correlation between lymph node involvement and the expression of HIF1a - the typical hypoxia marker. Those results stay in contrast to those published by Tawadros at al. [27] who observed a significant association between HIF1a expression and lymph node involvement in endometrial cancer. The explanation of our observation needs further studies

The FIGO stage at the time of diagnosis is an important prognostic factor in endometrial tumors, apart from the degree of cancer differentiation. Studies on the relationship between the expression of selected TLR's and VEGFR receptors and the clinical stage of the disease allowed us to demonstrate statistically significant relations. FIGO III and IV stage tumors are associated with significantly lower TLR3 expression, high VEGFR1 expression and low VEGFR2 expression. Similar relationships were not stated for TLR1, TLR2 and TLR4. The HIF1 a expression in relation to the FIGO stage did not present a statistically different relation.
The relations observed in our study might confirm the hypothesis indicating a significant role of VEGF through selected TLRs in the pathogenesis of endometrioid endometrial malignancies. Selected TLRs may influence the proliferation of a tumor by inducing a response that causes the process of inhibiting tumor progression. In this process, the receptor for epidermal growth factor (VEGFR) appears to be an important factor. The possibility of pharmacological intervention in the immune response to the pathogenic molecular factors that trigger this response may be a promising alternative to the treatment of endometrial malignant tumors. However, this hypothesis requires further research.

\section{Conflict of interest}

The authors declared no potential conflict of interest with respect to the research, authorship, and/or publication of this article.

\section{Ethical approval}

We declare that all experiments were performed in accordance with the current law of Poland. The investigations were approved by the Bioethical Commision of Medical University of Lodz (RNN/16/16/KE).

\section{REFERENCES}

1. Łuczak MW, Roszak A, Pawlik P, et al. Increased expression of HIF-1A and its implication in the hypoxia pathway in primary advanced uterine cervical carcinoma. Oncol Rep. 2011; 26(5): 1259-1264, doi: 10.3892/or.2011.1397, indexed in Pubmed: 21887475.

2. Yu Li, Wang L, Chen S. Endogenous toll-like receptor ligands and their biological significance. J Cell Mol Med. 2010; 14(11): 2592-2603, doi: 10.1111/j.1582-4934.2010.01127.x, indexed in Pubmed: 20629986.

3. Clarke DL, Davis NHE, Majithiya JB, et al. Development of a mouse model mimicking key aspects of a viral asthma exacerbation. Clin Sci (Lond). 2014; 126(8): 567-580, doi: 10.1042/CS20130149, indexed in Pubmed: 24152048.

4. Nadeem A, Siddiqui N, Al-Harbi NO, et al. TLR-7 agonist attenuates airway reactivity and inflammation through $\mathrm{Nrf2}$-mediated antioxidant protection in a murine model of allergic asthma. Int J Biochem Cell Biol. 2016; 73: 53-62, doi: 10.1016/j.biocel.2016.02.004, indexed in Pubmed: 26851512.

5. Netea MG, Van Der Graaf CAA, Vonk AG, et al. The role of toll-like receptor (TLR) 2 and TLR4 in the host defense against disseminated candidiasis. J Infect Dis. 2002; 185(10): 1483-1489, doi: 10.1086/340511, indexed in Pubmed: 11992285.

6. van der Heijden IM, Wilbrink B, Tchetverikov I, et al. Presence of bacterial DNA and bacterial peptidoglycans in joints of patients with rheumatoid arthritis and other arthritides. Arthritis Rheum. 2000;43(3): 593-598, doi: 10.1002/1529-0131(200003)43:3<593::AID-ANR16>3.0.CO;2-1, indexed in Pubmed: 10728753.

7. Whiteside TL. The tumor microenvironment and its role in promoting tumor growth. Oncogene. 2008; 27(45): 5904-5912, doi: 10.1038/onc.2008.271, indexed in Pubmed: 18836471.

8. Li H, Han Y, Guo Q, et al. Cancer-expanded myeloid-derived suppressor cells induce anergy of NK cells through membrane-bound TGF-beta 1. J Immunol. 2009; 182(1): 240-249, doi: 10.4049/jimmunol.182.1.240, indexed in Pubmed: 19109155.

9. Strauss L, Bergmann C, Whiteside TL. Human circulating CD4+CD25highFoxp3+ regulatory $T$ cells kill autologous CD8+ but not CD4+ responder cells by Fas-mediated apoptosis. J Immunol. 2009; 182(3): 1469-1480, doi: 10.4049/jimmunol.182.3.1469, indexed in Pubmed: 19155494. 
10. Yusuke S, Yasufumi G, Norihiko N, et al. $\mathrm{H}$ : Cancer Cells Expressing Toll-like Receptors and the Tumor Microenvironment. Cancer Microenvironment. 2009; 2(Suppl 1): 205-214.

11. Zhou M, McFarland-Mancini MM, Funk HM, et al. Toll-like receptor expression in normal ovary and ovarian tumors. Cancer Immunol Immunother. 2009; 58(9): 1375-1385, doi: 10.1007/s00262-008-0650-y, indexed in Pubmed: 19184006.

12. Dan HC, Sun M, Kaneko S, et al. Role of X-linked inhibitor of apoptosis protein in chemoresistance in ovarian cancer: possible involvement of the phosphoinositide-3 kinase/Akt pathway. Drug Resist Updat. 2002; 5(3-4): 131-146, doi: 10.1016/s1368-7646(02)00003-1, indexed in Pubmed: 12237081.

13. Kim WY, Lee JW, Choi JJ, et al. Increased expression of Toll-like receptor 5 during progression of cervical neoplasia. Int J Gynecol Cancer. 2008; 18(2): 300-305, doi: 10.1111/j.1525-1438.2007.01008.x, indexed in Pubmed: 17587322.

14. Lee JW, Choi JJ, Seo ES, et al. Increased toll-like receptor 9 expression in cervical neoplasia. Mol Carcinog. 2007; 46(11): 941-947, doi: 10.1002/mc.20325, indexed in Pubmed: 17440926.

15. Xu X, Yan Y, Xun Q, et al. Combined silencing of VEGF-A and angiopoietin-2, a more effective way to inhibit the Ishikawa endometrial cancer cell line. Onco Targets Ther. 2019; 12: 1215-1223, doi: 10.2147/OTT. S194064, indexed in Pubmed: 30863089.

16. Carmeliet P.VEGF as a key mediator of angiogenesis in cancer. Oncology. 2005; 69 Suppl 3: 4-10, doi: 10.1159/000088478, indexed in Pubmed: 16301830.

17. Li H, Han Y, Guo Q, et al. Cancer-expanded myeloid-derived suppressor cells induce anergy of NK cells through membrane-bound TGF-beta 1. J Immunol. 2009; 182(1): 240-249, doi: 10.4049/jimmunol.182.1.240, indexed in Pubmed: 19109155.

18. Lotze MT, Zeh HJ, Rubartelli A, et al. The grateful dead: damage-associated molecular pattern molecules and reduction/oxidation regulate immunity. Immunol Rev. 2007; 220: 60-81, doi: 10.1111/j.1600-065X.20 07.00579.x, indexed in Pubmed: 17979840.

19. Martins TM, Muniz CS, Andrade VB, et al. Changes in endometrial transcription of TLR2, TLR4, and CD14 during the first-week postpartum in dairy cows with retained placenta. Theriogenology. 2016; 85(7): 1282-1288, doi: 10.1016/j.theriogenology.2015.12.013, indexed in Pubmed: 26777563

20. Tian $Q$, Xue $Y$, Zheng $W$, et al. Overexpression of hypoxia-inducible factor $1 a$ induces migration and invasion through Notch signaling. Int J Oncol. 2015; 47(2): 728-738, doi: 10.3892/ijo.2015.3056, indexed in Pubmed: 26094772.

21. Allhorn S, Böing $C$, Koch AA, et al. TLR3 and TLR4 expression in healthy and diseased human endometrium. Reprod Biol Endocrinol. 2008; 6: 40, doi: 10.1186/1477-7827-6-40, indexed in Pubmed: 18775079.

22. Grimmig T, Moench R, Kreckel J, et al. Toll Like Receptor 2, 4, and 9 Signaling Promotes Autoregulative Tumor Cell Growth and VEGF/PDGF Expression in Human Pancreatic Cancer. Int J Mol Sci. 2016; 17(12), doi: 10.3390/ijms17122060, indexed in Pubmed: 27941651.

23. Gu CJ, Xie F, Zhang B, et al. High Glucose Promotes Epithelial-Mesenchymal Transition of Uterus Endometrial Cancer Cells by Increasing ER/GLUT4-Mediated VEGF Secretion. Cell Physiol Biochem. 2018; 50(2): 706-720, doi: 10.1159/000494237, indexed in Pubmed: 30308493.

24. Mahecha $A M$, Wang $H$. The influence of vascular endothelial growth factor-A and matrix metalloproteinase- 2 and -9 in angiogenesis, metastasis, and prognosis of endometrial cancer. Onco Targets Ther. 2017; 10: 4617-4624, doi: 10.2147/OTT.S132558, indexed in Pubmed: 29033580.

25. Wang J, Taylor A, Showeil R, et al. Expression profiling and significance of VEGF-A, VEGFR2, VEGFR3 and related proteins in endometrial carcinoma. Cytokine. 2014; 68(2): 94-100, doi: 10.1016/j.cyto.2014.04.005, indexed in Pubmed: 24845798.

26. Giatromanolaki A, Sivridis E, Brekken R, et al. The angiogenic ?vascular endothelial growth factor/flk-1(KDR) receptor? pathway in patients with endometrial carcinoma. Cancer. 2001; 92(10): 2569-2577, doi: 10.1002/1097-0142(20011115)92:10<2569::aid-cncr1609>3.0.co;2-3.

27. Tawadros Al, Khalafalla MM. Expression of programmed death-ligand 1 and hypoxia-inducible factor-1a proteins in endometrial carcinoma. J Cancer Res Ther. 2018; 14(Supplement): S1063-S1069, doi: 10.4103/0973-1482.202891, indexed in Pubmed: 30539847. 